Der Vorsitzende: Wir gehen nun zu Punkt 2 der Tagesordnung über und ich gebe Herrn Dr. Grünhut das Wort.

\title{
Die Verwendung der Mineralstoffe bei Backpulvern.
}

\section{Von \\ L. Grünhut-Wiesbaden.}

M. H.! Der folgende Bericht verwertet in der Hauptsache die Ergebnisse einer Experimentaluntersuchung, die von W. Fresenius und mir auf breiter Grundlage in Angriff genommen ist. Leider war es unmöglich, sie bis zu unserer heutigen Tagung abzuschließen, und so muß ich von vornherein darauf hinweisen, daß es noch unfertiges ist, was ich Thnen vortrage. Ich darf aber sogleich hinzufügen, daß unsere Arbeiten doch schon soweit vorgeschritten sind, daß sie bestimmte Richtlinien klar erkennen lassen und mich damit instandsetzen, ausreichend begründete Vorschläge zu vertreten und sie Ihnen zur Beschlußfassung zu empfehlen.

Backpulver sind Stoffe, die eine Lockerung des Teiges durch Gase herbeiführen, die - im Gegensatz zu den durch Sauerteig oder Hefe entwickelten - nicht durch Gärungsvorgänge, sondern durch rein chemische Umsetzungen entstehen. Am längsten bekannt sind in dieser Beziehung jene Mischungen, bei denen einerseits Natriumbydrocarbonat und auf der anderen Seite eine organische Säure oder das saure Salz einer solchen, und zwar meistens Weinsäure bezw. Weinstein, benutzt wurden.

Weinsäure und deren Salze lassen sich, bei der Kleinheit unserer Weinerzeugung, aus inländischen Rohstoffen 'nur in sehr beschränkten Mengen gewinnen, und Backpulver dieser Art können deshalb nicht mehr bereitet werden. So war es nötig, andere Mischungen heranzuziehen, und man hat dabei nicht nur an früher schon gelegentlich bewährtes angeknüpft, sondern vielfach auch ganz neue Bahnen betreten. Das, was nach der einen wie nach der anderen Seite hin seither in Erscheinung getreten ist, wollen wir nunmehr kritisch betrachten. Wir können hierbei zwei Gruppen unterscheiden: die Sulfat-Backpulver und die Phosphat-Backpulver.

Bei den Sulfat-Backpulvern handelt es sich um Mischungen, die als wirksame Bestandteile einerseits Natriumbydrocarbonat und auf der anderen Seite Alkalihydrosulfat oder Aluminiumsulfat oder Alaun enthalten. Die Kohlensäureentwickelung beruht bei ihnen also entweder auf der glatten Säurewirkung der Hydrosulfate oder auf der Umsekzung

$$
6 \mathrm{NaHCO}_{3}+\mathrm{Al}_{2}\left(\mathrm{SO}_{4}\right)_{3}=3 \mathrm{Na}_{2} \mathrm{SO}_{4}+2 \mathrm{Al}(\mathrm{OH})_{3}+6 \mathrm{CO}_{2} .
$$

Sie scheinen eine Frfindung der neuesten Zeit und erst im Kriege in größerem Umfange in Verkehr gebracht worden zu sein.

Ich kann mich nicht mit ihnen befreunden. Gegen die Alkalihydrosulfate spricht schon, daß sie rein technisch sich als wenig geeignet erweisen. Ihre sehr hygroskopische Beschaffenheit bereitet Schwierigkeiten aller Art, von der Gleichmäßigkeit der Vermischung angefangen bis zur Haltbarkeit der fertigen Ware. Dazu kommt als weiterer technischer Nachteil, daß sie wie starke Säuren wirken, sodaß die Umsetzung mit dem Natriumhydrocarbonat sich zu schnell vollzieht, um in vollem Um. fange der Teiglockerung zugute kommen zu können. Das bedenklichste aber ist, daß am SchluB beide Komponenten des Backpulvers, der hydrocarbonatalkalische und der saure, als einfaches Alkalisulfat zurückbleiben, d. h. als ein Stoff, der durch- 
aus nicht als indifferent gelten kann. $5 \mathrm{~g}$ Natriumbydrocarbonat, die man für $500 \mathrm{~g}$ Mehl gebraucht, liefern mit der äquivalenten Menge Natriumhydrosulfat eine Ausbeute von $8,46 \mathrm{~g}$ Natriumsulfat $\left(\mathrm{Na}_{2} \mathrm{SO}_{4}\right)$, entsprechend $19,2 \mathrm{~g}$ Glaubersalz $\left(\mathrm{Na}_{2} \mathrm{SO}_{4} \cdot 10 \mathrm{H}_{2} \mathrm{O}\right)$; letzteres aber verwendet man bereits in einer Dosierung von $1,0-2,5 \mathrm{~g}-$ die also schon in einem Gebäck aus 27-65. g Mehl enthalten sein könnte - als Laxans.

Auch gegen die Sulfat-Backpulver, die als sauer wirkenden Bestandteil Alaun oder A luminiumsulfat enthalten, bin ich nicht ohne Bedenken; zum mindesten so lange, bis nicht durch nene, den besonderen Verhältnissen nach Art und Menge der Verwendung angepaßte Versuche berufener Ärzte die Unschädlichkeit "dargetan ist. Bisher hat man Alaunzusätze zum Zweck der Wiederherstellung des Wasserbindungsvermögens verdorbenen Mehles ganz allgemein für unzulässig erklärt, und zwar nicht nur, weil sie die Verwendung schlechten Mehles ermöglichen, sondern auch deshalb, weil man sie für gesundheitsgefährlich hielt. Und dabei bewegten sich diese Zusätze um etwa $1,5 \mathrm{~g}$ Alaun auf je $500 \mathrm{~g} \mathrm{Mehl}$; bei Backpulvern hingegen wäre die $5 \mathrm{~g}$ Natriumhydrocarbonat äquivalente Menge, d. h. $9,4 \mathrm{~g}$ Alaun, $\mathrm{KAl}\left(\mathrm{SO}_{4}\right)_{2}: 12 \mathrm{H}_{2} \mathrm{O}$, erforderlich: mehr als das sechsfache. Schließlich ist auch hier, wie im vorigen Falle, die Bildung von Alkalisulfaten als Endumsetzungsprodukte zu beachten: ein Backpulver aus 5 g Natriumhydrocarbonat und der äquivalenten Menge Kalialaun ergibt 4,23 g Natriumsulfat $\left(\mathrm{Na}_{2} \mathrm{SO}_{4}\right)\left(=9,6 \mathrm{~g}\right.$ Glaubersalz) und 1,73 $\mathrm{g}$ Kaliumsulfat $\left(\mathrm{K}_{2} \mathrm{SO}_{4}\right)$, ein Backpulver aus $5 \mathrm{~g}$ Natriumbydrocarbonat und der äquivalenten Menge Aluminiumsulfat gibt gleichfalls $4,23 \mathrm{~g}$ Natriumsulfat $\left(\mathrm{Na}_{2} \mathrm{SO}_{4}\right)$.

Die Sulfat-Backpulver wären also, vom Standpunkt unseres heutigen Wissens, samt und sonders abzulehnen. Günstiger als sie schneiden die Phosphat-Backpulver vor unserem Urteil ab; nicht nur, daß - bei rationeller Mischung - keinerlei theoretisch-pharmakologische Einwände gegen sie zu erheben sind, vielmehr darf geradezu ibre Unschädlichkeit durch bis weit in die Friedensjahre zurückreichende und zum Teil sehr umfangreiche Benutzung als praktisch sichergestellt gelten.

In diesem Sinne wäre vor allem das seit mehr als 50 Jahren in Amerika außerordentlich weit verbreitete Horsford-Backpulver zu nennen, das aus einer Mischung: von Natriumbydrocarbonat und einem sog. "Säurepulver" besteht. Zur Bereitung des letzteren wurde Knochenasche mit Schwefelsäure zu Calciumdihydrophosphat, $\mathrm{Ca}\left(\mathrm{H}_{2} \mathrm{PO}_{4}\right)_{2}$, umgesetzt und das dabei sich ausscheidende, schwer lösliche Calciumsulfat durch Filtration abgetrennt. Nach Einengen zum Sirup krystallisierte das Phosphat aus; die Krystalle wurden, ưm sie auszutrocknen, mit Stärkemehl vermischt; diese Mischung war dann, nach dem Mahlen, das Säurepulver. - Liebig ist vielfach für die Verwendung dieses Backpulvers auch bei uns eingetreten. Er stellte fest, daß zur Lockerung des Teiges von $500 \mathrm{~g}$ Mehl 4,96 g Natriumbydrocarbonat erforderlich sind. Die Menge des Säurepulvers wird im genauen stöchiometrischen Verbältnis hierzu zugefügt. Außerdem empfabl Liebig noch die Zugabe der äquivalenten Menge Kaliumchlorid, um dem Organismus eine entsprechende Menge Kaliumsalze zuzuführen: ein Vorschlag, der, soweit ich weiß, weder damals, noch später größere Beachtung fand; nur wenige Fabriken nahmen bei uns die Herstellung auf. Erst in der letzten Zeit vor dem Kriege sind in Deutschland Phosphat-Backpulver, aber nach anderen Vorschriften, reichlicher bereitet worden; jetzt - im Kriege und im Zeichen der "Ersatzstoffe" - bilden sie, wegen des bereits erwähnten Weinsäuremangels, geradezu die Regel.

Sie bestehen samt und sonders aus Natriumbydrocarbonat und aus einem sauren 
Phosphat. Als letzteres wird meistens Calciumdihydrophosphat, $\mathrm{Ca}\left(\mathrm{H}_{2} \mathrm{PO}_{4}\right)_{2}$, in einer Reihe von Fallen aber auch Calciumhydrophosphat, $\mathrm{CaHPO}_{4}, 2 \mathrm{H}_{2} \mathrm{O}$, verwendet. Neuerdings wird dann noch Ammoniumdihydrophosphat, $\left(\mathrm{NH}_{4}\right) \mathrm{H}_{2} \mathrm{PO}_{4}$, benutzt. Zum Verständnis der ganzen Zusammenhänge scheint es mir dienlich, zunächst einmal theoretisch die Zusammensetzung derartiger Mischungen, sowie der von ihnen in Gebäck hinterlassenen Mineralstoffe klarzulegen. Ich gehe dabei von dem schon mehrfach herbeigezogenen Grundwert: $5 \mathrm{~g}$ Natriumbydrocarbonat in einer für $500 \mathrm{~g}$ Mehl bestimmten Packung aus, den Liebig begründete und der von den besseren. Her. stellern auch heute noch inne gehalten wird.

Aus diesen $5 \mathrm{~g}$ werden $2,62 \mathrm{~g}$ Kohlensäure $\left(\mathrm{CO}_{2}\right)$ entwickelt, die ibrerseits bei $100^{\circ}$ - der Temperatur, die das Innere des Brotes in dem auf $200-270^{\circ}$ angeheizten Backofen erreicht - eine Raumerfüllung von rund $1800 \mathrm{ccm}$ haben. Das erscheint auf den ersten Anblick als ein reichlich großer Betrag; man erkennt aber rasch seine Berechtigung, wenn man sich vergegenwärtigt, daß im allgemeinen Gebäcke von $500 \mathrm{~g}$ lufttrockenem Weizenmehl das Volumen von $2000 \mathrm{ccm}$, Gebäcke aus $500 \mathrm{~g}$ lufttrockenem Roggenmehl $1500 \mathrm{ccm}$ Rauminhalt nicht unterschreiten sollen, damit die Lockerung genüge.

Legt man zunächst einmal die Vorstellung zugrunde, es vermöchten sich die genannten sauren Phosphate mit Natriumhydrocarbonat glatt zu Kohlendioxyd und tertiären Phosphaten umzusetzen, so würde das folgende Reaktionsgleichungen bedingen :

1. $\mathrm{NaHCO}_{3}+\mathrm{CaHPO}_{4}=\mathrm{CaNaPO}_{4}+\mathrm{CO}_{2}+\mathrm{H}_{2} \mathrm{O}$.

2. $4 \mathrm{NaHCO}_{3}+\mathrm{Ca}\left(\mathrm{H}_{2} \mathrm{PO}_{4}\right)_{2}=\mathrm{CaNa}_{4}\left(\mathrm{PO}_{4}\right)_{2}+4 \mathrm{CO}_{2}+4 \mathrm{H}_{2} \mathrm{O}$.

3. $3 \mathrm{NaHCO}_{3}+\left(\mathrm{NH}_{4}\right) \mathrm{H}_{2} \mathrm{PO}_{4}=\mathrm{Na}_{3} \mathrm{PO}_{4}+\mathrm{NH}_{3}+3 \mathrm{CO}_{2}+3 \mathrm{H}_{2} \mathrm{O}$.

Diesen Gleichungen entsprächen folgende Vorschriften für die Normalpackung für $500 \mathrm{~g} \mathrm{Mehl,} \mathrm{unter} \mathrm{der} \mathrm{Voraussetzung} \mathrm{der} \mathrm{Benutzung} \mathrm{chemisch} \mathrm{reiner} \mathrm{Salze:}$
I. $5,00 \mathrm{~g} \mathrm{NaHCO}_{3}$

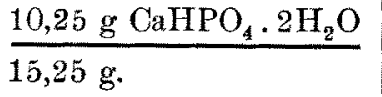
II. $5,00 \mathrm{~g} \mathrm{NaHCO}$
III. 5,00 g $\mathrm{NaHCO}_{3}$

$\frac{3,48 \mathrm{~g} \mathrm{Ca}\left(\mathrm{H}_{2} \mathrm{PO}_{4}\right)_{2}}{8,48 \mathrm{~g} .}$
$2,28 \mathrm{~g}\left(\mathrm{NH}_{4}\right) \mathrm{H}_{2} \mathrm{PO}_{4}$
$7,28 \mathrm{~g}$.

Bereitet man nun derartige Mischungen und bestimmt darin die wirksame, d. h. die durch Kochen mit Wasser auszutreibende Kohlensäure, so findet man in allen Fällen weit weniger, als nach den aufgestellten Gleichungen zu erwarten ist. Die Reaktion schreitet eben nicht bis zum tertiären Phosphat vor, sie kommt vielmehr, schon beim sog. sekundären, dem Monohydrophosphat zum Stehen. Statt jener drei Gleichungen sind demnach die folgenden zugrunde zu legen:

4. $2 \mathrm{NaHCO}_{3}+\mathrm{CaHPO}_{4}=\mathrm{Na}_{2} \mathrm{HPO}_{4}+\mathrm{CaCO}_{3}+\mathrm{CO}_{2}+\mathrm{H}_{2} \mathrm{O}$.

5. $2 \mathrm{NaHCO}_{3}+\mathrm{Ca}\left(\mathrm{H}_{2} \mathrm{PO}_{4}\right)_{2}=\mathrm{Na}_{2} \mathrm{HPO}_{4}+\mathrm{CaHPO}_{4}+2 \mathrm{CO}_{2}+\mathrm{H}_{2} \mathrm{O}$.

6. $2 \mathrm{NaHCO}_{3}+\left(\mathrm{NH}_{4}\right) \mathrm{H}_{2} \mathrm{PO}_{4}=\mathrm{Na}_{2} \mathrm{HPO}_{4}+\mathrm{NH}_{3}+2 \mathrm{CO}_{2}+2 \mathrm{H}_{2} \mathrm{O}$,

und ihnen entsprechen die folgenden Mischungen reiner Salze:

\begin{tabular}{|c|c|c|}
\hline $\begin{array}{l}\text { IV. } 5,00 \mathrm{~g} \mathrm{NaHCO}_{3} \\
\quad 5,12 \mathrm{~g} \mathrm{CaHPO} \\
\end{array}$ & $\begin{aligned} & 5,00 \mathrm{~g} \mathrm{NaHCO} \\
& 6,97 \mathrm{~g} \mathrm{Ca}\left(\mathrm{H}_{2} \mathrm{PO}_{4}\right)_{2} \\
&\end{aligned}$ & $\begin{array}{ll}\text { VI. } 5,00 \mathrm{~g} \mathrm{NaHCO}_{3} \\
3,42 \mathrm{~g}\left(\mathrm{NH}_{4}\right) \mathrm{H}_{2} \mathrm{PO}_{4} \\
\end{array}$ \\
\hline $10,12 \mathrm{~g}$ & $11,97 \mathrm{~g}$. & $8,42 \mathrm{~g}$. \\
\hline
\end{tabular}

Mit Beziehung auf Gleichung 4, bezw. Vorschrift IV ist hervorzubeben, dap also das Calciummonohydrophosphat überhaupt nicht als Säure wirkt, und daß in Backpulvern solcher Art; die von uns übrigens tatsächlich im Handel gefun- 
den wurden, nur die sog. doppelt gebundene Kohlensäure zur Wirkung kommt, also nur die Hälfte der im Natriumbydrocarbonat enthaltenen. D ie wäre nun schließlich, wenn auch sehr langsam, auch ohne jeden weiteren Zusatz bei der Backofentemperatur auszutreiben, aber das Phosphat beschleunigt das sichtlich. Und abgesehen davon kommt ihm noch eine andere Bedeutung zu. Ohne dasselbe bliebe im Gebäck am Ende Natriumcarbonat $\left(\mathrm{Na}_{2} \mathrm{CO}_{3}\right)$ zurück: ein höchst unerwünschter Bestandteil wegen seines. laugigen Geschmacks und seiner alkalischen Reaktion, welch' letztere überdies die Farbe des Gebäckes recht ungünstig beeinflussen kann. Ist aber Calciummonohydrophosphat zugegen, dann tritt an Stelle des Natriumearbonats das Carbonat des Calciums, das keinen der genannten Schäden hervorbringt. $\mathrm{Zweckmäfig}$ ist trotzdem eine solche Zusammensetzung nicht; es geht ja bei ihr die Hälfte der Kohlensäure für die Triebarbeit des Backpulvers verloren.

Ich brauche kaum hinzuzufügen, daß jedes praktisch lösliche Calciumsalz dasselbe leisten kann, wie das Calciummonohydrophosphat, beispielsweise das $\mathrm{S}$ ulfat: $2 \mathrm{NaHCO}_{3}+\mathrm{CaSO}_{4}=\mathrm{Na}_{2} \mathrm{SO}_{4}+\mathrm{CaCO}_{3}+\mathrm{CO}_{2}+\mathrm{H}_{2} \mathrm{O}$.

A ber selbstverständlich ist das Calciumsulfat als Kohlensäureentwickler praktisch noch weniger geeignet als das Monohydrophosphat, weil es gleichfalls die eben exwähnten Nachteile besitzt und dazu noch den weiteren mit sich bringt, im fertigen Gebäck Natriumsulfat zu hinterlassen.

Die Vorschriften IV, V und VI, die ich oben für mehr oder minder rationelle Backpulvermischungen errechnet habe, sind aber zum Teil noch nicht diejenigen, die der Wirklichkeit entsprechen. Denn die Fabrikationspraxis verwendet in der Regel keine chemisch reinen Salze, sondern benutzt vielfach technische Präparate. Wir haben uns eine Reihe von solchen aus Backpulverfabriken beschafft und sie analysiert und dabei gefunden, daß nur das Ammoniumdihydrophosphat rein im Sinne des Chemikers war. Das untersuchte Präparat enthielt

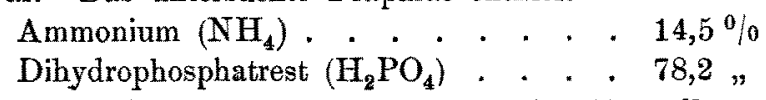

Der Rest von 7,3\% war wohl Feuchtigkeit oder Krystallwasser.

Ganz anders waren die analysierten Calciumphosphate beschaffen; wir fanden in dreien derselben, einem Monophosphat und zwei Dihydrophosphaten, folgende $\mathrm{Zu}$ sammensetzung:

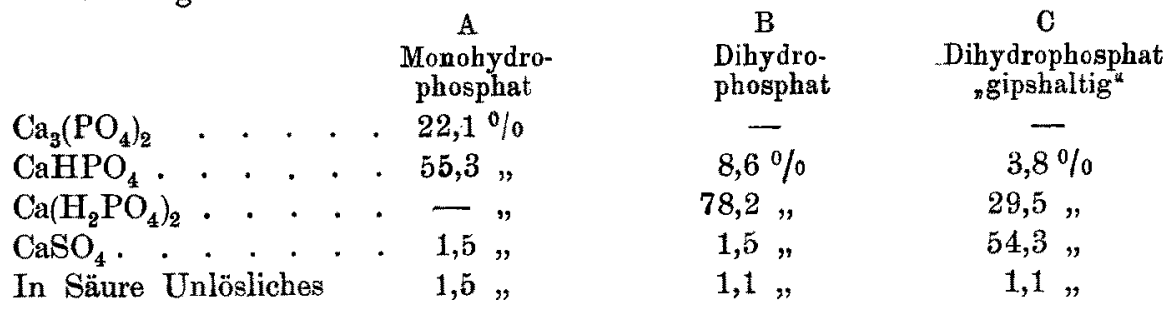

Die Analysen wurden, im Anschluß an das mabanalytische Verfabren von B. Pfyl, so ausgeführt, daß einmal die Gesamt-Alkalität gegen Methylorange und zum anderen der Gesamt-Phosphatrest $\left(\mathrm{PO}_{4}\right)$ ermittelt, und danach die Verteilung auf die verschiedenen Aciditätsstufen vorgenommen wurde. Außerdem wurde der Sulfatrest $\left(\mathrm{SO}_{4}\right)$ gewichtsanalytisch bestimmt und auf $\mathrm{CaSO}_{4}$ umgerechnet. - Alle Präparate enthalten also neben dem Phosphat, nach dem sie benannt sind, auch solches von der geringeren Aciditätsstufe und überdies Calciumsulfat. Die Menge desselben ist 
in $A$ und $B$ nur gering; beide sind offenbar nach dem Doppelsuperphosphatverfahren hergestellt; das sehr gipsreiche Präparat $\mathrm{C}$ ist hingegen sichtlich nach Art der gewöhnlichen. Superphosphate bereitet.

Berechnet man nun in gleicher Weise wie zuvor die stöchiometrische Zusammensetzung von Backpulvern, die derartige Erzeugnisse enthalten, so kommt man zu folgenden Vorschriften:

VII. 5,00 g $\mathrm{NaHCO}_{3}$

\section{7,33 g Phosphat A}

$12,33 \mathrm{~g}$.
VIII. $5,00 \mathrm{~g} \mathrm{NaHCO}_{3}$ 8,92 g Phosphat B 13,92. g.
IX. 5,00 g $\mathrm{NaHCO}_{3}$ 23,63 g Phosphat $\mathrm{C}$ $28,63 \mathrm{~g}$.

Ein Blick auf die Vorschriften IV-IX lehrt, daß das Gewich einer für $500 \mathrm{~g}$ Mehl bestimmten Backpulverpackung schon allein innerhalb des Rahmens dieser Erfahrungen zwischen 8,4 und $28,6 \mathrm{~g}$ schwanken kann. Und ebenso groß und mannigfaltig sind die Unterschiede in den Mineralstoffen, die nach erfolgtem Trieb von diesen Pulvern in dem aus $500 \mathrm{~g} \mathrm{Mehl} \mathrm{erzeugten} \mathrm{Gebäck} \mathrm{zurückbleiben.}$ Sie sind so, wie sie sich rechnerisch ergeben, in der folgenden Tafel zusammengestellt:

Es bleiben zurück aus Mischung:

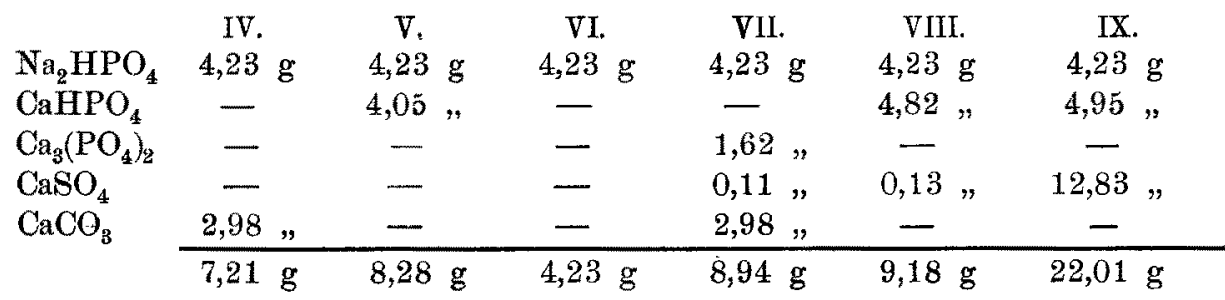

Die mit Monohydrophosphaten bereiteten Mischungen (IV und VII) sind aus den vorhin angegebenen Gründen (S. 39) als unrationell zu bezeichnen und scheiden für die weitere Betrachtung aus. Von den übrigen darf wohl in Beziehung auf IX gesagt werden, daß es für das Gebäck bezw. den dasselbe Verzehrenden einen solchen Ballast von Mineralstoffen mitbringt (22. $\mathrm{g}$, darunter $12,8 \mathrm{~g}$ Calciumsulfat), daß die Verwendung so gipshaltiger Erzeugnisse wie $\mathrm{C}$ im Backpulvergewerbe zum mindesten als höchst unerwünscht bezeichnet werden muß.

So bleiben noch V, VI und VIII übrig, und da drängt sich unwillkürlich die Frage auf, ob nicht die so viel geringere Mineralstoff-Belastung, die das Ammoniumdibydrophosphatbackpulver VI gegenüber den Calciumdihydrophosphatbackpulvern V und. VIII dem Gebäck auferlegt, derartigen Erzeugnissen einen wesentlichen Vorzug vor jenen anderen verleiht. Sie zu beantworten, kann nicht meine Aufgabe sein; sie gehört zur Zuständigkeit des Arztes, der sie an der Hand von Feststellungen über die pharmakologische Wirkung der Phosphate auf den Organismus zu beantworten hätte.

Nun ist aber auch das, was zuletzt für die Handelspräparate errechnet wurde, immer noch Theorie, und die Praxis ist weit entfernt, sich mit Vorschriften, wie die hier unter IV bis VIII errechneten zu begnügen; sie fügt vielmehr dem Natriumhydrocarbonat und dem Phosphat ausnahmslos noch andere St of fe hinzu, die mit der Kohlensäureentwickelung, mit der Triebkraft des Pulvers, gar nichts zu tun haben. Früher war das meistens Stärkemehl, das ich ja schon als Bestandteil des Horsfor d-Pulvers erwähnen konnte, oder auch Getreidemeh1; jetzt, wo diese beiden der 
amtlichen Verteilung unter dem Brotmarkenregime unterstellt sind, benutzt man ausschließlich mineralische Zusätze: Kreide, Gips, Magnesiumcarbonat, Bolus. Thnen gegenüber Stellung zu nehmen, muß heute unsere Hauptaufgabe sein.

Das kann wohl heute als sicber gelten, daß diese Zusätze in der Regel nicht deshalb erfolgen, um mit ihnen die Ware zu strecken, sondern daf sie geschehen, um eine technische Notwendigkeit zu erfüllen. Die verwendeten Phosphate sind samt und sonders bygroskopisch und die sie enthaltenden Backpulver ziehen beim Aufbewahren in den üblichen Papierbeutelpackungen schon auf dem Lager, dann später beim Zwischenhändler und zuletzt im Haushalt, Feuchtigkeit an. Damit ist aber zugleich den Bestandteilen die Möglichkeit zur gegenseitigen Umsetzung gegeben: die Kohlensäureentwickelung beginnt, und die. Triebkraft der Mischung geht, je länger um so mehr zurück. Das sicherste Mittel hiergegen wäre die Abfassung des Natriumhydrocarbonats und des Phosphats in zwei gesonderte Beutel, bezw. Hülsen, nach Art der Brausepulver. Versuche nach dieser Richtung. sind früher schon mit dem Horsford-Pulver gemacht worden und haben zu wenig günstigen Ergebnissen geführt; auch die heutigen Fabrikanten widersireben einer solchen. Maßnahme, die sie wohl mit Recht als unpraktisch bezeichnen und gegen deren verbindliche Einführung überdies noch die Papiernot spricht. So bleibt denn nur der Ausweg übrig, durch Zumischung eines chemisch unwirksamen Stoffes die Reaktion auch im feucht gewordenen Backpulver, gewissermaßen durch Zwischenlagerung zwischen die wirksamen Bestandteile, hintanzuhalten; das ist der Sinn der Zusätze, die man um deswillen heute häufig als „Treanungs mit tel " bezeichnet.

Die rationellsten Trennungsmittel sind selbstverständlich Kartoffelmehl oder Getreidemehl; sie sind normale Bestandteile dès Teiges, und mit ihnen wird dem Gebäck keinerlei fremdgeartetes zugebracht. Und so möchte ich, wenn wir dann das Ergebnis dieser Besprechưng zu Beschlüssen formen, Ihnen in erster Linie einen solchen zur Anuahme empfehlen; der besagt, daß wir die Freigabe von Mehl oder Stärkemehl an die Backpulverfabriken zur Benutzung als Trennungsmittel für äußerst wünschenswert halten.

Solánge solcher Wunseh noch nicht erfüllt ist, wird die Backpulverindustrie der 'mineralischen Trennungsmittel nicht evitraten könñen, und wir müssen deshalb die bisher benutzten kritisch auf ihre Eignung prüfen und die Mindestmenge festsetzen, die ausreicht, den Zweck zu 'erfüllen.

Da ihre Aufgabe eine rein mechanische ist: sie sollen die eigentlich wirksamen Stoffe so weit voneinander isolieren, daß sie auch im feuchten Zustande nicht aufeinander reagieren, so liegt die Vermutung nahe, dab ihre Eignung zu ihrer Lockerheit in Beziebung steht. Wir haben, um hierüber einen Begriff zu erhalten, das spezifisché Einrüttelungśolumen einiger der in der Praxis verwendeten Stoffe ermittelt; d. h. die Raumerfüllung bestimmt; die $\mathbf{~} \mathrm{g}$ beim Einrütteln in ein Hohlmaß einnimmt. Die Versuche wurden so angestellt, daß ein tarierter Zylinder, der bei 50 ecm eine Marke trug, unter Einrütteln bis zur Marke mit dem Pulver gefüllt und dann gewogen wurde. So ergab sich:

Spezifisches

Einrüttelungswolumen.

Gemahlene Kreide .

$0,70 \mathrm{ccm}$

Gemahlener Gips, ungebrannt . . . 0,84 "

Magnesiumcarbonat . . . . . . . 1,32"

Gefälltes Calciumcarbonat . . . . 2,06 " 
Die gefundenen Werte sind natürlich nich t absolute Konstanten der betreffenden Stoffe, sie werden vielmehr von Präparat zu Präparat je nach Mahlungsgrad und sonstiger mechanischer Bearbeitung in recht weiten Grenzen schwanken können. Aber sie zeigen doch, daß Unterschiede bestehen, und lehren, welche Größenordnungen in Betracht kommen können, und besonders bemerkenswert erscheint in dieser Beziehung die Verschiedenheit zwischen der gemahlenen Kreide und dém gefällten Calciumcarbonat. Aber auf der anderen Seite haben unsere bisherigen Versuche den hieraus zu erwartenden Untersehied in der Eignung als Trennungsmittel nicht erkennen lassen.. Vergleichsmischungen desselben Backpulvers, einerseits mit der gemahlenen Kreide und andererseits mit dem gefällten Calciumcarbonat verhielten sich beim Aufbewahren in einer feuchten Kammer nicht merklich voneinander verschieden. Dieser Mißerfolg kann freilich noch durch die Versuchsanordnung bedingt sein: wir hatten die Mischungen durch Zusammenreiben in der Reibschale hergestellt, und da ist es nicht unwahrscheinlich, da: unter dem Druck des Pistills die Unterschiede in der Loekerheit aufgehoben wurden. Wir sind im Begriff, die betreffenden Versuche unter Ausschlub solcher Möglichkeit zu wiederholen.

Ich gehe nunmehr die einzelnen mineralischen Trennungsmittel der Reihe nach durch und beginne mit dem weipen Bolus. Er ist ein Erzeugnis des Mineralreichs, das in die Klasse der Tone gehört undrin seinen reineren Abarten nahezu reines Aluminiumbydrosilikat ist. Bolus ișt offizinell, und das Deutsche Arzneibuch verzeichnet ganz bestimmte Anforderungen an seine Reinheit. Therapeutisch wird er bei Cholera, Brechdürchfall, Meteorismus und Rachendiphtherie angewendet. Man reicht ihn teelöffelweise in Aufschwemmungen mit der doppelten Menge Wassers und gibt Erwachsenen täglich $50-100 \mathrm{~g}$, Kindern $30 \mathrm{~g}$ und Säuglingen $15 \mathrm{~g}$. Im ärztlichen Schriftum ist ausdrücklich ausgesprochen, daß seine vollkommene Indifferenz sichergestellt sei und daß er in beliebigen Mengen innerlich verabreicht werden könne.

Ich bin mir wobl bewubt, und habe das auch immer yertreten, daß aus der medizinischen Anwendung eines Stoffes im allgemeinen keine Schlüsse auf seine unbeschränkte Verwendung im Lebensmittelgewerbe gezogen werden dürfen, denn es ist ein anderes, ob man ihn gelegentlich als Heilmittel, und ein anderes, ob man ihn regelmäBig in der Nahrung zu sich nimmt. Aber im vorliegenden Sonderfall, beim Bolus, scheint mir eine Ausnahme vorzuliegen. Wenn die Unschädlichkeit bei so gewaltigen Dosen, wie ich sie Ihnen nannte, erwiesen ist, wenn selbst Säuglinge anstandslos $15 \mathrm{~g}$ an einem Tage vertragen, dann dạf man ihn gewiß für vollkommen harmlos halten und Mengen von $2-3 \mathrm{~g}$, wie sie mit dem Backpulver in eine $500 \mathrm{~g} \mathrm{Mehl} \mathrm{entsprechende} \mathrm{Gebäckmenge} \mathrm{gelangen,} \mathrm{auch} \mathrm{bei} \mathrm{täglichem}$ Verzehr als sicher unschädlich erachten. Ich vermag also keinen Grund zu erblicken, der unş veranlassen müßte, gegen seine Verwendung als Trennungsmittel vorzugehen; vorausgesetzt natürlich, daß er rei $n$ ist, was am besten an Häd der Arzneibuchvorschrift zu prüfen wäre.

Minder indifferent ist bereits das Calciumcarbouat, weil es - unverändert im Gebäck zurückbleibend - nach der Aufnahme in dep Organismus geeignet ist, die Acidität des Magensaftes herabzusetzen. Man hat, um das zu verhindern, erwogen, ob dem Calciumcarbonat nicht vielleicht eine etwas veränderte Rolle im Backpulver zugewiesen werden könne, derart, daß es nicht nur als Trennungsmittel, sondern gleichzeitig auch als Kohlensäurequelle dient; d. h. daß es beim Anrühren des Teiges mit zersetzt wird. Das wäre nur möglich, wenn man dem Backpulver zugleich eine äqui- 
valente Menge Natriumdihydrophosphat hinzufügte; das hat sich aber, wie die Fabrikanten glaubwürdig versichern, wegen der ganz besonders hohen Hygroskopizität dieses Salzes nicht praktisch verwirklichen lassen. Die endgültige Stellungnahme gegenüber dem Calciumcarbonat im Backpulver muß -unter diesen Umständen dem ärztlichen Sachverständigen überlassen bleiben; er allein kann darüber abschließend urteilen, ob sein erwähnter Einfluß auf den" Verdauungsvorgang innerhalb der Grenzen, die sich bei seiner Verwendung ganz von selbst ergeben, eine wirkliche Gefährdung oder gàr Schädigung bedeutet. Soweit man heute sehen kann, und angesichts der Tatsache, $d a ß$, von Vielen erdige oder alkalische Tafelwässer regelmäßig getrunken werden, scheint der Körper doch ziemlich duldsam gegen Verminderungen der Magensaftacidität zu sein, und so glaube ich, wird man den Calciumcarbonatzusatz in kleinen Mengen als einen Notbehelf in der Backpulverfabrikation vorläufig dulden

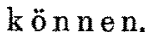

Für das Magnesiumcarbonat gelten dieselben Erwägungen wie für das Calciumcarbonat; man wird für dieses den gleichen Standpunkt einräumen rü̈ssen wie für jenes.

Weniger duldsam stehe ich dem Calciumsulfat gegenüber. Wir treffen es als Bestandteil der regelmäßigen Nahrung zuweilen im Trinkwasser an: es gibt Orte, auch solche mit zentraler Wasserversorgung, die sich kein anderes Wasser als eines mit hoher bleibender Härte beschaffen können. Unmittelbare Gesundheitsschädigungen, die man früher damit in Verbindung brachte, sind bei sorgfältiger Oberprüfung durch berufene Hygieniker nicht bestätigt worden; aber übrig geblieben ist das absprechende Urteil gegen solche.Wässer wegen ibres schlechten Geschmacks. Und dieser schlechte Geschmack tritt - darüber besteht Übereinstimmung im Gebäck, das mit einem calciumsulfatreichen Backpulver hergestellt ist; noch viel unerquicklicher hervor, und daraus allein scheint mir ein ausreichender Grund hervorzugehen, die Zulässigkeit von Calciumsulfat als Trennungsmittèl abzulehnen. Man kann das um so eher, als ja den Fabrikanten im Bolus, im Calcium. und in Magnesiumcarbonat ausreichende Hilfsstoffe zur Verfügung stehen. Und zu alledem kommt noch hinzu, daß in einem Gebäck, das mit calciumsulfathaltigem Pulver hergestellt ist, Sulfation und Natriumion (letzteres aus dem Hydrocarbonat stammend) nebeneinander zugegen sind, sodaß es nicht ausgeschlossen ist, daß es die unliebsamen Wirkungen des Glaubersalzes hervorzubringen vermöchte.

Die Ablehnung des Calciumsulfates bezieht sich nicht bloß auf seine Verwendung als Trennungsmittel, sie gilt auch gegenüber jedem anders gearteten Hineinbringen in das Backpulver. Die Benutzung gipsreicher Calciumphosphate, wie sie nach dem Superphosphatverfahren gewonnen werden, halte ich demnach für unzulässig. Die geringen Mengen Gips, die auch in die nach dem Doppel superphosphatverfahren bereiteten Phosphate als technisch unvermeidbare Beimengungen übergehen, wird man hingegen dulden können und dulden müssen. Der gleiche Standpunkt der Duldung gilt schließlich auch gegenüber terti.ärem Calciumphosphat, sofern es als technisch unvermeidbare Verunreinigung in den Backpulver-Rohstoffen von Haus aus enthalten ist.

Sind wir so mit Beziehung auf die $\Delta \mathrm{rt}$ der zulässigen mineralischen Trennungsmittel zu -einem Urteil gelangt, so müssen wir uns auch noch eine Meinung über die Menge bilden, in der sie noeh als einwandfrei angesehen werden können. Man darf dazu wohl ganz allgemein, sagen, sie soll so groß sein dürfen, wie es die 
Praxis nötig hat, aber auch nicht größer. Um hierfür Unterlagen zu schaffen, haben W. Fresenius und ich gleichfalls Versuche angestellt, derart, daß wir verschiedene Mischungen mit wechselnden Mengen der Trennungsmittel versetzten, sie, in Papierbeutel verpackt, in feuchte Kammern brachten und ihr Verhalten hierin untersuchten.

Als feuchte Kammern dienten uns große Zinkblechglocken von 62,5 Liter Fassung, die mit ihrem unteren Rand in einer ölgefüllten Rinne standen, sodab ihr Inneres völlig dicht von der AuBenluft abgeschlossen war. Unter diese Glocken stellten wir Schalen mit Kaliumcarbonatlösung von bestimmten Sättigungsstufen, aus deren Dampfspannung, die aus einer Arbeit von $W$ üllner entnommen werden konnte, die relative Feuchtigkeit der Luft unter der Glocke berechnet werden konnte. In drei parallelen Versuchsarten benutzten wir drei verschiedene Lösungen, denen relative Feuchtigkeiten von 50 bezw. 75 bezw. 90\% entsprachen. Unter jeder Glocke war überdies noch eine Drabthürde aufgestellt, auf' die die Papierbeutel mit den Mustern ausgelegt wurden.

Die Versuche sind zur Zeit erst mit zwei Grundmischungen in Angriff genommen, die aus den oben mit $A$ und $B$ bezeichneten Calciumphosphaten (S. 40) hergestellt worden waren und zwar aus ersterem nach der auf $\mathrm{S}$. 39 mitgeteilten Reaktionsgleichung 1, aus letzterem nach der Gleichung 2, nach Vorschriften also, die wir mittlerweile (S. 39) bereits als praktisch minder vorteilhaft erkannt haben. Natürlich sind andere. Versuche nach anderen Rezepten bereits eingeleitet; doch können auch diese schon zeigen, worum es sich handelt.

Die folgende Tafel I gibt zunächst die Gewichtsveränderungen wieder, die die Pulver nach 2-, 9- und 16-tägigem Aufenthalt unter der Glocke erfuhren. Die Zahlen zeigen die Veränderungen in Hundertteilen des Anfangsgewichtes an, Gewichtsabnahmen sind durch negatives, Gewichtszunahmen durch positives Vorzeichen gekennzeichnet. Die vorgesetzten Ziffern 1, 2, 3 bezeichnen den Aufenthalt in Luft von 50 bezw. 75 bezw. $90 \%$ relativer Feuchtigkeit.

Tafel I.

\begin{tabular}{|c|c|c|c|c|c|c|c|c|c|}
\hline Bezeichnung & & 2 Tage & 9 Tage & 16 Tage & Bezeichuung & & 2 Tage & 9 Tage & 16 Tage \\
\hline $\begin{array}{c}\mathrm{X} \\
5 \mathrm{~g} \mathrm{NaHCO} \\
14,74 \mathrm{~g} \mathrm{Monohydro}- \\
\text { phosphat A }\end{array}$ & $\begin{array}{l}1 \\
2 \\
3\end{array}$ & $\begin{array}{l}-1,7 \\
-0,5 \\
+1,4\end{array}$ & $\begin{array}{l}-2,0 \\
-1,2 \\
-0,02\end{array}$ & $\begin{array}{l}-1,4 \\
-1,6 \\
+0,4\end{array}$ & $\begin{array}{c}\text { XI. } \\
5 \mathrm{~g} \mathrm{NaHCO} \\
4,28 \mathrm{~g} \mathrm{Dihydro-} \\
\text { phosphat B }\end{array}$ & $\begin{array}{l}1 \\
2 \\
3\end{array}$ & $\begin{array}{r}-1,5 \\
+2,1 \\
+8,4\end{array}$ & $\begin{array}{r}-1,5 \\
+3,3 \\
+20,6\end{array}$ & $\begin{array}{r}-1,3 \\
+9,3 \\
+19,1\end{array}$ \\
\hline
\end{tabular}

In der folgenden Tafel II sind die Ergebnisse von Parallelversuchen wiedergegeben, bei denen diesen Grundmischungen Trennungsmittel _- und zwar gemahlene Kreide, gefälltes Calciumcarbonat, Magnesiumcarbonat. und gemahlener ungebrannter Gips - hinzugefügt waren. Es wurden sowohl Versuchsreihen angestellt, bei denen das Trennungsmittel $10 \%$ als auch solche, bei denen es $20 \%$ der Gesamtmasse betrug. 
Tafel II.

Tafel III.

\begin{tabular}{|c|c|c|c|c|c|c|c|c|c|}
\hline Mischung & & $2 \mathrm{Tag}$ & Tage & 16 Tage & Mischung & & 2 Tage & 9 Tage & 16 Tage \\
\hline $\begin{array}{l}90 \text { Tle. } X . \\
10 \text { Tle. gemahlene } \\
\text { Kreide }\end{array}$ & $\begin{array}{l}1 \\
2 \\
3\end{array}$ & $\begin{array}{l}-1,1 \\
-1,6 \\
-1,6\end{array}$ & $\begin{array}{l}-1,8 \\
-4,7 \\
+0,7\end{array}$ & $\begin{array}{l}-2,2 \\
-6,0 \\
+0,6\end{array}$ & $\begin{array}{l}90 \text { Tle. XI. } \\
10 \text { Tle. gemahlene } \\
\text { Kreide }\end{array}$ & $\begin{array}{l}1 \\
2 \\
3\end{array}$ & $\begin{array}{r}-1,6 \\
+2,2 \\
+6,1\end{array}$ & $\begin{array}{r}-1,4 \\
+2,0 \\
+16,6\end{array} \mid$ & $\begin{array}{r}-1,3 \\
+7,7 \\
+16,8\end{array}$ \\
\hline $\begin{array}{l}90 \text { Tle. } \mathbf{x} . \\
10 \text { Tle. gefälltes } \\
\text { Calciumearbonat }\end{array}$ & $\begin{array}{l}1 \\
2 \\
3\end{array}$ & $\begin{array}{l}-1,2 \\
-1,8 \\
-3,0\end{array}$ & $\begin{array}{l}-1,8 \\
-5,2 \\
-1,8\end{array}$ & $\begin{array}{l}-2,1 \\
-6,3 \\
-1,3\end{array}$ & $\begin{array}{l}90 \text { Tle. XI. } \\
10 \text { Tle. gefälltes } \\
\text { Calciumearbonat }\end{array}$ & $\begin{array}{l}1 \\
2\end{array}$ & $\begin{array}{r}-1,9 \\
+2,6 \\
+5,2\end{array}$ & $\begin{array}{r}-2,0 \\
+2,2 \\
+16,2\end{array} \mid$ & $\begin{array}{r}-1,5 \\
+2,2 \\
+13,5\end{array}$ \\
\hline $\begin{array}{l}90 \text { Tle. } X . \\
10 \text { Tle. Magnesium- } \\
\text { carbonat }\end{array}$ & $\begin{array}{l}1 \\
2 \\
3\end{array}$ & $\begin{array}{r}-0,9 \\
-0,3 \\
-1,7\end{array}$ & $\begin{array}{r}-1,4 \\
-3,4 \\
+1,5\end{array}$ & $\begin{array}{l}-1,3 \\
-5,1 \\
+1,6\end{array}$ & $\begin{array}{c}90 \text { T'le. XI. } \\
10 \text { Tle. Magnesium- } \\
\text { carbonat }\end{array}$ & $\begin{array}{l}1 \\
2 \\
3\end{array}$ & $\begin{array}{r}-1,6 \\
+2,4 \\
+6,3\end{array}$ & $\begin{array}{r}-1,8 \\
+3,5 \\
+17,9\end{array}$ & $\begin{array}{r}-0,8 \\
+8,8 \\
+18,1\end{array}$ \\
\hline $\begin{array}{l}90 \text { T'le. X. } \\
10 \text { Tle. gemahlener } \\
\text { ungebrannter Gips }\end{array}$ & $\begin{array}{l}1 \\
2 \\
3\end{array}$ & $\begin{array}{r}-1,3 \\
-1,9 \\
+0,2 \\
\end{array}$ & $\begin{array}{r}-2,0 \\
-5,0 \\
-0,05 \\
\end{array}$ & $\begin{array}{l}-2,0 \\
-5,8 \\
-0,7\end{array}$ & $\begin{array}{c}90 \text { Tle. XI. } \\
10 \text { Tle. gemahlener } \\
\text { ungebrannter Gips }\end{array}$ & $\begin{array}{l}1 \\
2 \\
3\end{array}$ & $\begin{array}{r}-3,4 \\
+0,9 \\
+7,7 \\
\end{array}$ & $\begin{array}{r}-4,0 \\
+4,6 \\
+18,1 \\
\end{array}$ & $\begin{array}{r}-3,4 \\
+6,1 \\
+17,4\end{array}$ \\
\hline $\begin{array}{l}80 \text { Tle. } X . \\
20 \text { Tle. gemahlene } \\
\text { Kreide }\end{array}$ & $\begin{array}{l}1 \\
2 \\
3\end{array}$ & $\begin{array}{r}-0,7 \\
-0,4 \\
+0,9\end{array}$ & $\begin{array}{l}-1,2 \\
-1,9 \\
-1,7\end{array}$ & $\begin{array}{l}-1,8 \\
-3,7 \\
-1,6\end{array}$ & $\begin{array}{l}80 \text { Tle. XI. } \\
20 \text { Tle. gemahlene } \\
\text { Kreide }\end{array}$ & $\begin{array}{l}1 \\
2 \\
3\end{array}$ & $\begin{array}{r}+2,5 \\
+1,5 \\
+4,4\end{array}$ & $\left|\begin{array}{r}+2,1 \\
+5,2 \\
+12,3\end{array}\right|$ & $\begin{array}{r}-2,2 \\
-7,3 \\
+12,6\end{array}$ \\
\hline $\begin{array}{l}80 \text { Tle. X. } \\
20 \text { Tle, gefalltes } \\
\text { Calciumearbonat }\end{array}$ & $\begin{array}{l}1 \\
2 \\
3\end{array}$ & $\begin{array}{r}-0,9 \\
-0,1 \\
+1,5\end{array}$ & $\begin{array}{l}-1,3 \\
-1,0 \\
-2,2\end{array}$ & $\begin{array}{l}-1,7 \\
-4,3 \\
-4,4\end{array}$ & $\begin{array}{l}80 \text { Tle. XI. } \\
20 \text { Tle. gefälltes } \\
\text { Calciumcarbonat }\end{array}$ & $\begin{array}{l}1 \\
2 \\
3\end{array}$ & $\begin{array}{r}-0,7 \\
+6,0 \\
+2,9\end{array}$ & $\begin{array}{r}-1,1 \\
+6,8 \\
+11,0\end{array}$ & $\begin{array}{r}-2,5 \\
+6,3 \\
+11,6\end{array}$ \\
\hline $\begin{array}{l}80 \text { Tle. } \mathrm{X} . \\
20 \text { Tle. Magnesium- } \\
\text { carbonat }\end{array}$ & $\begin{array}{l}1 \\
2 \\
3\end{array}$ & $\begin{array}{r}-0,2 \\
+0,1 \\
+1,3\end{array}$ & $\begin{array}{l}-1,4 \\
-0,6 \\
+0,9\end{array}$ & $\begin{array}{l}-1,9 \\
-1,8 \\
+0,5\end{array}$ & $\begin{array}{l}80 \text { Tle. XI. } \\
20 \text { Tle. Magnesinm } \\
\text { carbonat }\end{array}$ & $\begin{array}{l}1 \\
2 \\
3\end{array}$ & $\begin{array}{l}-0,7 \\
+1,6 \\
+3,7\end{array}$ & $\begin{array}{l}-1,1 \\
+2,9 \\
+14,4\end{array}$ & $\begin{array}{l}-1,8 \\
+2,8 \\
+15,1\end{array}$ \\
\hline $\begin{array}{l}80 \text { Tle. X. } \\
20 \text { Tle. gemahlener } \\
\text { angebrannter Gips }\end{array}$ & $\begin{array}{l}1 \\
2 \\
3\end{array}$ & $\begin{array}{r}-1.1 \\
-0,6 \\
+1,2\end{array}$ & $\begin{array}{r}-1,9 \\
-2,0 \\
+2,0\end{array}$ & $\begin{array}{l}-2,0 \\
-3,3 \\
+1,2\end{array}$ & $\begin{array}{c}80 \text { Tle. XI. } \\
20 \text { Tle. gemahlener } \\
\text { ungebrannter Gips }\end{array}$ & $\begin{array}{l}1 \\
2 \\
3\end{array}$ & $\begin{array}{r}-2,6 \\
+1,5 \\
+4,4\end{array}$ & $\begin{array}{r}-4,3 \\
+3,7 \\
+12,8\end{array}$ & $\begin{array}{r}-5,0 \\
+4,4 \\
+14,2\end{array}$ \\
\hline
\end{tabular}

Fine Betrachtung dieser Zahlen lehrt, daß an minder feuchter Luft (1 und 2) meistens Gewichtsabnahmen, bei sehr feuchter Luft (3) Gewichtszunahmen zu verzeichnen sind. Offenbar hat man es aber überall mit der Resultante zweier Vorgänge zu tun: Gewichtszunahme infolge Wasseraufnahme und Gewichtsabnahme infolge Kohlensäureentwickelung aus der feucht gewordenen Masse. Welcher Anteil in jedem Fall auf das eine, welcher auf das andere fällt, das wird man erst sagen können, wenn auch Kohlensäurebestimmungen an den in den feuchten Kammern ausgesetzten Proben ausgeführt worden sind. Das ist bereits in die Wege geleitet; heute schon verwertbare Ergebnisse liegen aber noch nicht vor.

Bei diesem unabgeschlossenen Stande kann zur Zeit füglich auf eine Einzelbesprechung der in den Tafeln niedergelegten Ergebnisse noch verzichtet werden. Doch sei immerhin folgendes hervorgehoberi: Die Mischung $\mathbf{X}$ ist, sowohl für sich als nach Zugabe von Trennungsmitteln, sichtlich unempfindlicher als die Misehung XI; es ist also das in letzterer enthaltene Dihydrophosphat B viel hygroskopischer als das Monohydrophosphat A. Die Eignung in bezug auf Luftbeständigkeit ist also beim Dihydrophosphat geringer, während es um seiner chemischen Reaktionen willen wesentliche Vorzüge besitzt. Aber ich glaube nicht, daß man, um seiner geringeren Luftbe- 
ständigkeit willen, sich nun doch dazu entschließen sollte, es hinter das Monohydrophosphat zurückzustellen. Des letzteren oben auseinander gesetzte Nachteile können durch seine größere Beständigkeit nicht aufgehoben werden.

Die Versuche in Tafel II im Vergleich mit Tafel I zeigen ferner, daß beim Calciumdihydrophosphat-Backpulver die Unbeständigkeit durch einen Gehalt an $10 \%$ Trennungsmittel noch nicht sehr erheblich, durch einen. Gehalt an 20\% schon merklicher vermindert wird.

Soweit sind wir mit unseren Versuchen gelangt. Wir sind bestrebt, sie in einer Weise weiterzuführen, die etwa folgende Regelung erlauben wird: Wenn man einerseits festsetzt, dab eine für $500 \mathrm{~g}$ Mehl bestimmte Backpulverpackung 2,6 $\mathrm{g}$ wirksame - $\mathrm{d}$. $\mathrm{h}$. beim Kochen mit Wasser fortgehende - Kohlensäure enthalten muß (S. 39), und wenn man andererseits festsetzt, daß eine solche Packung ein bestimmtes Höchstgewicht nicht überschreiten darf, dann ist einem Übermaß an Trennungsmittel der Riegel vorgeschoben und zugleich eine Form der Begrenzung gewählt, die die praktische Überwachung durch die Organe der Nahrungsmittelaufsicht verbältnismäßig einfach gestaltet. Zur Begründung brauche ich nur darauf hinzuweisen, daß - wie die vorhergehenden Betrachtungen über die geeigneten Rezepte erwiesen - für jene $2,6 \mathrm{~g}$ Kohlensäure ein bestimmtes Gewicht an Natriumhydrocarbonat und Phosphat unbedingt erforderlich und demnach, bei fesigelegtem Gesamt-Gewicht, der Spielraum für alle anderen Zusätze eingeschränkt ist.

$\mathrm{Zu}$ ergänzen wäre diese Festsetzung dadurch, daß - um der Veränderlichkeit der Ware Rechnung zu tragen - für die wirksame Kohlensäure ein Spielraum von $\pm 10 \%$ ihrer Menge zugelassen werden mub, daß also die Werte von 2,35-2,85\% zulässig sind (auf das Backpulvergewicht gerechnet). - Eine weitere Ergänzung besteht dann noch in einer Aufzählung der zulässigen Trennungsmittel. Das sind nach meiner Auffassung: in erster Linie Mehl und Kartoffelmehl, und dann erst - und nur solange die eben genanten nicht freigegeben sind - Calciumcarbonat, Magnesiumcarbonat und reiner weiBer Bolus.

Bis man über die nötigen Erfabrungen verfügt, um in solchem Sinne vorzugehen, halte ich es für unbedenklich, die genannten Trennungsmittel in einem Betrage, der insgesamt $20 \%$ des Backpulvergewichtes nicht überschreitet, zuzulassen.

Der Vorsitzende: Ich bitte nun, Herrn Professor Wirthle, sein Referat zu erstatten.

\section{Über Backpulver.}

\section{Berichterstatter: Prof. Dr. F. Wirthle-Würzburg.}

Im Laufe des Weltkrieges haben in den Verkaufsstellen für Nahrungsmittel die Vorräte ganz bedeutend abgenommen, ja manche Waren sind vollständig verechwunden. Backpulver findet man aber auch heute noch selbst in kleinen Geschäften, und gar nicht selten in verschiedenen Arten. Da diese Erzeugnisse zur Bereitung von Nahrungsmitteln Verwendung finden und letztere erst durch die treibende Kraft dieser Backhilfsmittel genußfähig werden, ist es unsere Pflicht, auch den Backpulvern unsere 